\title{
Embryo Morphokinetic Parameters Evaluated Using a Time-Lapse Embryo Monitoring System Can Predict the Embryo Developmental Potential in ICSI Cycles
}

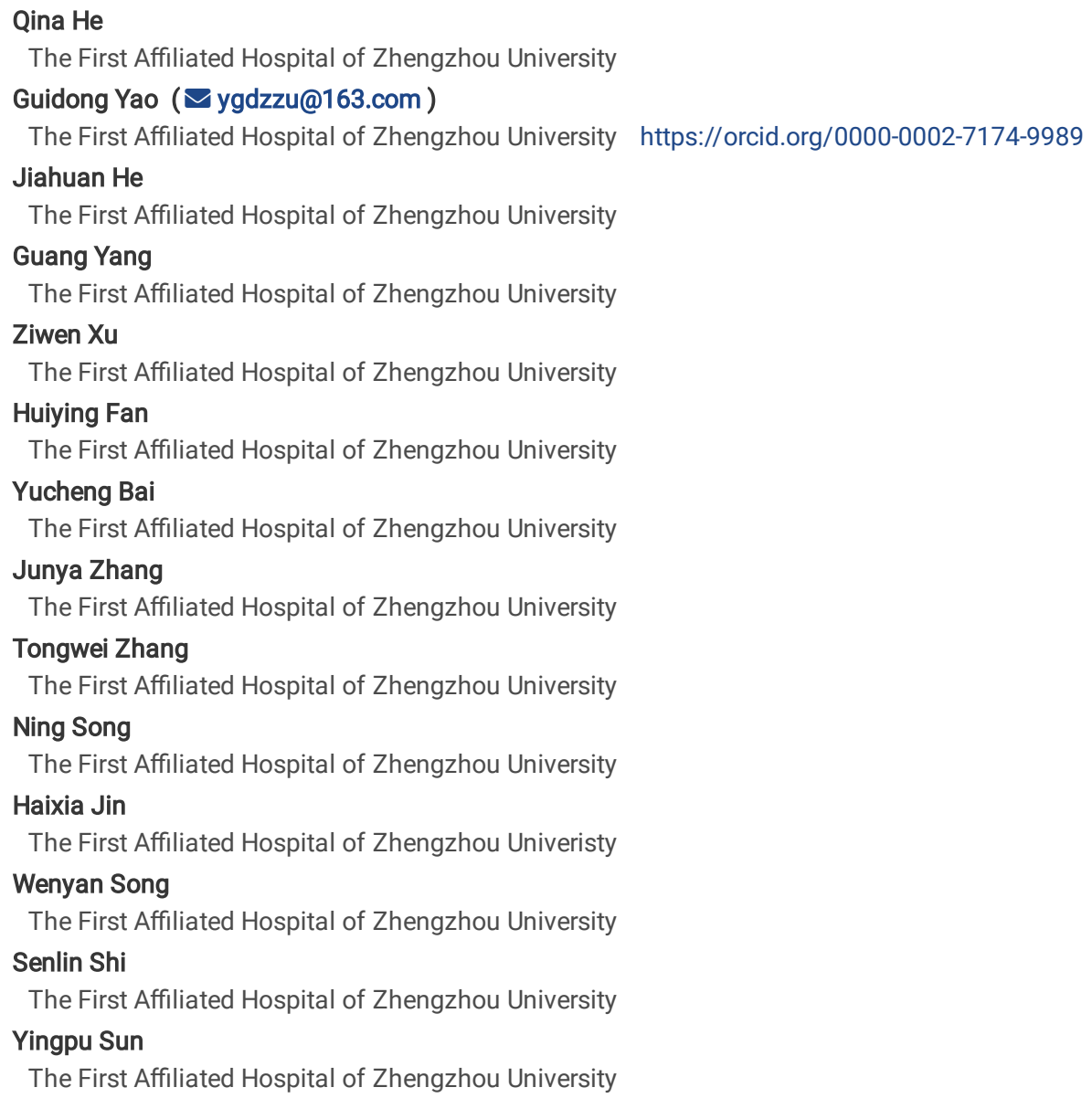




\section{Abstract}

At present, embryo morphology assessment based on the observation of the embryonic morphological characteristics at several specific time points has been mainly used for selecting the high-quality embryo. However, we all know that embryo development is a dynamic process. Many research results on the correlation between the embryo morphokinetic parameters and embryo quality and development potential were inconsistent. With the help of time-lapse imaging, the development processes and outcomes of a total of 365 embryos were cultured and analyzed in this study. The results showed that the mean tPNf and t2 of the high-quality embryo were significantly shorter than the low-quality embryo; the mean t2PBe and tPNa of the high-quality embryo from the implantation group were significantly shorter than those from the non-implantation group. In addition, based on the quartile grouping of each morphokinetic parameter, the embryos that had $21.15 \leq \mathrm{tPNf} \leq 25.30$ value were significantly higher in embryo quality when compared with the embryos that had the tPNf values outside the range on Days 3 . Similarly, the embryos that had values of $t 2 \leq 25.60$ were significantly higher in embryo quality than those with outside the range values on Days 3. Thus, we demonstrated that the morphokinetic parameter evaluated using a time-lapse embryo monitoring system can predict the embryo quality, and be benefit for the selection of the high-quality embryos and improvement for the implantation success of the patient in assisted reproductive technologies.

\section{Introduction}

Assisted reproductive technology (ART) has been advanced rapidly in recent decades, and tens of thousands of infertile couples have obtained offspring with the aid of ART. Selecting a single best-quality embryo for transfer, improving the implantation rate, and reducing the multiple pregnancy outcomes have been the intense focus of research in the field of ART. At present, standard embryo morphology assessment has been used for selecting the high-quality embryo for transfer, and this conventional method is mainly based on the observation of the fertilized embryo and its cleavage at several specific time points during the development, and then the embryos are scored using these morphological characteristics ${ }^{[1]}$. However, such evaluations have limitations ${ }^{[2]}$, because scoring may be affected by the subjective effects of the embryologists, and embryos need to be temporarily removed from the incubator during the assessment, which may cause stress to the embryo and negatively affect the embryo development. In addition, embryo development is a dynamic process, and embryo morphology at some time points does not fully reflect the entire process of embryo development.

In recent years, time-lapse monitoring system (TLM) was widely used in the embryology laboratory. The TLM is a technology, and it can continuously monitor the morphokinetic parameters of the early embryo during its development. Further. the embryo assessment can be performed without removing the embryos from the incubators using the TLM system. Previous time-lapse studies indicated that the embryo morphokinetic parameters obtained using TLM have certain predictive significance on the potential of blastocyst formation, implantation after embryo transfer, and live birth rate ${ }^{[3-6]}$. Aguilar et al. (2014) showed that the time of second polar body (2PB) extrusion and the time of pronuclear fading have a certain value in predicting embryo implantation rate ${ }^{[7]}$. However, some studies believed that embryo morphokinetic parameters were not related to the embryo developmental potential $\left.{ }^{8}, 9\right]$, and Barberet et al. (2019) believed that the time of second polar body extrusion and pronuclear fading were not related to the early embryo development ${ }^{[10]}$. The variations of these results may have been caused by the differences in the sample size, fertilization methods used, embryo culture conditions adopted, and characteristics of the research participants.

Therefore, the TLM was used to observe and record the embryos' development process from the intracytoplasmic sperm injection (ICSI) cycles in this study. We retrospectively analyzed the relationship between the identified embryo morphokinetic parameters such as the time of second polar body extrusion (t2PBe), the time of pronuclear appearance (tPNa), the time of pronuclear fading (tPNf), and the time of cleavage to 2-cells (t2), and the embryo quality, embryo developmental potential, and pregnancy outcome. The aims of this study were to explore whether the embryo morphokinetic parameters could predict the embryo development potential in the ICSI cycle and determine whether these morphokinetics variables can serve as effective evaluation indicators in embryo selection for improving embryo implantation rate and pregnancy rate.

\section{Materials And Methods Study design}

This study included 365 embryos involving 55 women (one cycle/person) who underwent ICSI treatment at the Center for Reproductive Medicine, the First Affiliated Hospital of Zhengzhou University. The inclusion criteria for this study were the female patients who underwent the first ICSI cycle, $\leq 35$ years of age, and only male infertility factors including oligozoospermia, asthenozoospermia, and obstructive azoospermia. Women with the abnormal uterine condition (hysteromyoma, endometriosis, etc.) and recurrent abortion were excluded. GERI time-lapse system (Genea Biomedx, Sydney, NSW, Australia)was used to culture the zygotes which were fertilized using the ICSI procedure. Furthermore, the embryo morphokinetic parameters were recorded and analyzed, including the times taken for second polar body extrusion (t2PBe), pronuclear appearance (tPNa), and pronuclear fading (tPNf), and cleavage to 2-cells (t2). ICSI and embryo culture

Transvaginal ultrasound-guided follicles' aspiration was performed $36 \mathrm{~h}$ after recombinant human chorionic gonadotropin (rhCG) administration. Oocytes retrieved were cultured for 1-2 hours before denudation in G-IVF plus medium (Vitrolife Sweden AB, Goteborg, Sweden). Then, the oocyte denudation was carried out using hyaluronidase (Vitrolife Sweden AB) andere cultured continuously in the G-IVF plus medium for 1-2 hour until the intracytoplasmic sperm injection. Ejaculated sperm and epididymal sperm were washed with SpermGrad gradient solution (Vitrolife) to obtain progressive motile sperm, whereas testicular sperm were obtained shredding the testicular tissue in the G-IVF plus medium using a $1 \mathrm{~mL}$ syringe needle and then washed directly with the G-IVF plus medium. Under an inverted microscope (TE2000-U, Nikon, Chiyoda, Japan), mature oocytes were fertilized using the ICSI procedure and transferred into the time-lapse culture dishes containing G-1 plus medium (Vitrolife) after the ICSI. All zygotes were incubated at $37^{\circ} \mathrm{C}$ and under the microenvironment 
containing $5.0 \% \mathrm{O} 2,6.0 \% \mathrm{CO} 2$, and $89.0 \% \mathrm{~N} 2$ controlled by the GERI time-lapse incubator. The images were automatically acquired at intervals of 10 min through the time-lapse monitoring system. From the fertilization until Day 3, embryos were selected to transfer or freeze or continuously be cultured until the blastocyst stage. For the blastocyst culture, the Day 3 embryos were transferred to the $\mathrm{G}-2$ plus medium (Vitrolife) and cultured at $37^{\circ} \mathrm{C}$ and under $5.0 \%$ O2, $6.0 \% \mathrm{CO} 2$, and $89.0 \% \mathrm{~N} 2$.

\section{Embryo scoring and embryo transfer}

Embryo morphology was only evaluated on Day 3 based on Peter's embryo scoring criteria (the number of blastomeres, percentage of fragmentation, and presence of multi-nucleation) ${ }^{[11]}$, and grades $₫$ and $\otimes$ were defined as the high-quality embryo. Blastocysts (on Day 5 or Day 6 ) were graded according to Gardner's criteria ${ }^{[12]}$ and the high-quality blastocysts were accordingly grouped (stage 3-6, AA, AB, BA, or BB). At the blastocyst stage, a single high-quality embryo on Day 5 was selected for embryo transfer. Embryo morphology scoring was performed by two trained embryologists. A total of 54 embryos were transferred to 46 patients. The number of embryos transferred was normally two, but in some cases, one embryo was transferred because of embryo quality.

The serum $\beta$-hCG value was determined 14 days after the embryo transfer. The $\beta$-hCG of $\geq 50 \mathrm{mlU} / \mathrm{mL}$ was defined as a biochemical marker for a positive pregnancy. The pregnancy was also confirmed by ultrasound examination when a gestational sac with a fetal positive heartbeat was visible after 35 days of the embryo transfer.

\section{Statistical analysis}

All statistical analyses were performed using the SPSS v.20.0 (IBM SPSS software, Chicago, IL, USA). The Student t-test was used to analyze the data. The continuous variables were presented as mean \pm standard deviation (SD), and the categorical variables were presented as percentages (\%). Furthermore, timing variables were converted from continuous variables into categorical variables. They were divided into groups based on their quartiles. An optimal range was defined as the combined range spanned over the two quartiles with the highest implantation rate. The categorical variables were analyzed using Chi-square tests. The P-value $<0.05$ was considered to be significant.

\section{Results}

\section{Characteristics of the enrolled patients}

In total, this retrospective analysis has utilized clinical data from 55 ICSI treatment cycles. The embryonic development data of 365 fertilized two pronuclear (2PN) zygotes from the time-lapse system recordings were used to analyze. In this current study, the mean age was $29.4 \pm 4.3$ years, the mean body mass index (BMI) was $22.0 \pm 2.9 \mathrm{~kg} / \mathrm{m} 2$, the mean number of oocytes retrieved was $12.4 \pm 3.4$, the mean value for anti-Müllerian hormone (AMH) was $3.47 \pm 2.3$ $\mathrm{ng} / \mathrm{mL}$, the mean value for estradiol(E2)was $6.86 \pm 2.0 \mathrm{mlU} / \mathrm{mL}$, and the mean value for luteinizing hormone (LH) was $5.32 \pm 2.73 \mathrm{mIU} / \mathrm{mL}$.

The morphokinetics of the embryo during the early development monitored using a time-lapse monitoring system (TLM) in the ICSI cycle

The time of monitoring the embryonic developmental events such as the second polar body extrusion, pronuclear appearance, pronuclear fading, and the cleavage of fertilized oocytes to 2-cells after ICSI using the TLM system, is shown in Fig. 1. For all evaluated embryos in the present study, the time interval from the ICSI to the second polar body extrusion(t2PBe)was $2.85 \pm 0.9 \mathrm{~h}$, the time interval from the ICSI to pronuclear appearance (tPNa) was $6.43 \pm 1.41 \mathrm{~h}$, the time interval from the ICSI to pronuclear fading (tPNf) was $23.36 \pm 3.08 \mathrm{~h}$, and the time interval from the ICSI to the cleavage of the fertilized oocyte to 2 cells embryo (t2) was $26.45 \pm 4.11 \mathrm{~h}$.

\section{Comparison of embryo morphokinetic parameters between high-quality and low-quality embryos}

According to Peter's embryo assessment system on cleavage stage, all Day 3 embryos from the 2PN zygotes were divided into the high-quality group and low-quality group. The embryo morphokinetic parameters were compared between the two groups and were shown in Table 1 . In the high-quality embryo group, the tPNf $(22.92 \pm 2.68$ vs. $24.33 \pm 3.69, \mathrm{P}<0.05)$ and the $22(25.58 \pm 2.74$ vs. $28.41 \pm 5.76, P<0.05)$ were found to be significantly shorter compared with the low-quality group. No significant differences for the variables t2PBe and tPNa were found between the groups (Pख0.05).

Table 1

Comparison of embryo morphokinetic parameters between the high-quality and low-quality embryos

\begin{tabular}{|llllll|}
\hline Group & $\mathbf{N}$ & t2PBe(h) & tPNa(h) & tPNf(h) & t2(h) \\
\hline High-quality & 238 & $2.80 \pm 0.79$ & $6.39 \pm 1.26$ & $22.92 \pm 2.68$ & $25.58 \pm 2.74$ \\
\hline Low-quality & 109 & $2.95 \pm 1.1$ & $6.56 \pm 1.75$ & $24.33 \pm 3.69$ & $28.41 \pm 5.76$ \\
\hline T & -1.379 & -1.048 & -3.976 & -6.151 \\
\hline P & 0.169 & 0.295 & $\varangle 0.05$ & $\varangle 0.05$ \\
\hline * The Pख0.05 was considered statistically significant. & \\
\hline
\end{tabular}

Relationship between embryo morphokinetic parameters and high-quality embryo ratio, and the blastocyst formation rate 
To explore the relationship between embryo morphokinetic parameters during early development and the high-quality embryos ratio and blastocyst formation rate, the Day 3 embryos were divided into groups based on the quartiles of each morphokinetic parameter, and the high-quality embryos ratio were compared among the groups (Table 2). For each timing variable, an optimal range for the high-quality embryo distribution was defined as the two consecutive quartiles with the highest high-quality embryo ratio (numbers in bold and underlined in the table). The results showed that the high-quality embryo rate was statistically different when compared with each tPNf quartile group, and when compared with each t2 quartile group $(P<0.05)$. Among them, the high-quality embryo rate was highest (73.4\%) when the tPNf was $22.83 \mathrm{~h}-25.3 \mathrm{~h}$, and when the $\mathrm{t} 2<24.0 \mathrm{~h}$ and no significant differences for highquality embryo rate were found for the other parameter quartile groups (Pख0.05).

Table 2

The relationship between embryo morphokinetic parameters and high-quality embryo rate

\begin{tabular}{|c|c|c|c|c|c|c|c|c|c|c|}
\hline \multirow{2}{*}{$\begin{array}{l}\text { Morphokinetic } \\
\text { parameters }\end{array}$} & \multicolumn{2}{|l|}{1} & \multicolumn{2}{|l|}{2} & \multicolumn{2}{|l|}{3} & \multicolumn{2}{|l|}{4} & \multirow{2}{*}{$x^{2}$} & \multirow[t]{2}{*}{$P$} \\
\hline & $\begin{array}{l}\text { Time } \\
\text { interval/h }\end{array}$ & $\begin{array}{l}\text { Rate of High- } \\
\text { quality } \\
\text { embryo(\%) }\end{array}$ & $\begin{array}{l}\text { Time } \\
\text { interval/h }\end{array}$ & $\begin{array}{l}\text { Rate of High- } \\
\text { quality } \\
\text { embryo(\%) }\end{array}$ & $\begin{array}{l}\text { Time } \\
\text { interval/h }\end{array}$ & $\begin{array}{l}\text { Rate of High- } \\
\text { quality } \\
\text { embryo(\%) }\end{array}$ & $\begin{array}{l}\text { Time } \\
\text { interval/h }\end{array}$ & $\begin{array}{l}\text { Rate of } \\
\text { High-quality } \\
\text { embryo(\%) }\end{array}$ & & \\
\hline t2PBe & $\otimes 2.25$ & 65.5 & $\begin{array}{l}2.25- \\
2.70\end{array}$ & 67.8 & $\begin{array}{l}2.70- \\
3.30\end{array}$ & 69.0 & $\varangle 3.30$ & 65.8 & 0.522 & 0.914 \\
\hline tPNa & $₫ 5.60$ & 67.0 & $\begin{array}{l}5.60- \\
6.25\end{array}$ & 60.6 & $6.25-7.0$ & 68.1 & $\otimes 7.0$ & 53.2 & 3.789 & 0.285 \\
\hline tPNf & $\otimes 21.15$ & 67.0 & $\begin{array}{l}21.15- \\
22.83\end{array}$ & 66.0 & $\begin{array}{l}22.83- \\
25.30\end{array}$ & 73.4 & $\otimes 25.30$ & 42.6 & 22.465 & $\varangle 0.001$ \\
\hline t2 & $\bowtie 24.0$ & 73.4 & $\begin{array}{l}24.0- \\
25.60\end{array}$ & 67.0 & $\begin{array}{l}25.60- \\
28.20\end{array}$ & 67.0 & $\otimes 28.20$ & 40.4 & 27.091 & $\varangle 0.001$ \\
\hline
\end{tabular}

To further explore the relationship between the embryo morphokinetic parameters and the blastocyst formation rate, the blastocyst formation rate was compared among the morphokinetic parameters quartile groups and was shown in Table 3. The results revealed that the blastocyst formation rate was not statistically different $(\mathrm{P}>0.05)$ among the morphokinetic parameters quartile groups.

Table 3

The relationship between the embryo morphokinetic parameters and the blastocyst formation rate

\begin{tabular}{|c|c|c|c|c|c|c|c|c|c|c|}
\hline \multirow{2}{*}{$\begin{array}{l}\text { Morphokinetic } \\
\text { parameters }\end{array}$} & \multicolumn{2}{|l|}{1} & \multicolumn{2}{|l|}{2} & \multicolumn{2}{|l|}{3} & \multicolumn{4}{|l|}{4} \\
\hline & $\begin{array}{l}\text { Time } \\
\text { interval/h }\end{array}$ & $\begin{array}{l}\text { Rate of } \\
\text { Blastocyst } \\
\text { Formation(\%) }\end{array}$ & $\begin{array}{l}\text { Time } \\
\text { interval } / \mathrm{h}\end{array}$ & $\begin{array}{l}\text { Rate of } \\
\text { Blastocyst } \\
\text { Formation(\%) }\end{array}$ & $\begin{array}{l}\text { Time } \\
\text { interval/h }\end{array}$ & $\begin{array}{l}\text { Rate of } \\
\text { Blastocyst } \\
\text { Formation(\%) }\end{array}$ & $\begin{array}{l}\text { Time } \\
\text { interval/h }\end{array}$ & $\begin{array}{l}\text { Rate of } \\
\text { Blastocyst } \\
\text { Formation(\%) }\end{array}$ & $\chi^{2}$ & $\mathbf{P}$ \\
\hline t2PBe & $\bowtie 2.25$ & 66.7 & $\begin{array}{l}2.25- \\
2.70\end{array}$ & 82.1 & $\begin{array}{l}2.70- \\
3.30\end{array}$ & 81.8 & $\otimes 3.30$ & 72.7 & 3.173 & 0.366 \\
\hline $\mathrm{tPNa}$ & $\otimes 5.60$ & 67.6 & $\begin{array}{l}5.60- \\
6.25\end{array}$ & 86.7 & $6.25-7.0$ & 78.9 & $\otimes 7.0$ & 70.3 & 4.054 & 0.256 \\
\hline tPNf & $₫ 21.15$ & 87.1 & $\begin{array}{l}1.15- \\
22.83\end{array}$ & 75.9 & $\begin{array}{l}22.83- \\
25.30\end{array}$ & 70.3 & $\varangle 25.30$ & 71.1 & 3.257 & 0.354 \\
\hline t2 & $\otimes 24.0$ & 90.6 & $\begin{array}{l}24.0- \\
25.60\end{array}$ & 71.4 & $\begin{array}{l}25.60- \\
28.20\end{array}$ & 70.3 & $\varangle 28.20$ & 71.1 & 5.201 & 0.158 \\
\hline
\end{tabular}

The influence of the patient's BMI status on embryos' morphokinetics during early development

Based on the Chinese body mass index (BMI) criteria formulated by the National Health and Family Planning Commission of the People's Republic of China, there are group $A(B M I<18.5 \mathrm{~kg} / \mathrm{m} 2)$, group $B(18.5 \mathrm{~kg} / \mathrm{m} 2 \leq \mathrm{BMI}<24 \mathrm{~kg} / \mathrm{m} 2)$ and group $\mathrm{C}(24 \mathrm{~kg} / \mathrm{m} 2 \leq \mathrm{BMI} \leq 32 \mathrm{~kg} / \mathrm{m} 2)$. We found that the high-quality embryos ratio, blastocyst formation, and high-quality blastocysts rate were different in participants with different BMI (Table 4). For the Day 3 embryos, the ratio of high-quality embryos in group $A$ is higher than that in the other groups, but no statistical differences were found. Besides, no differences for the blastocyst formation rate and the high-quality blastocyst rate were also found among the groups $(P>0.05)$. 
Table 4

The influence of the BMI status on early embryonic development

\begin{tabular}{|lllll|}
\hline BMI & N & Rate of High-quality Embryo(\%) & Rate of Blastocyst Formation(\%) & Rate of High-quality Blastocyst(\%) \\
\hline A (₫18.5) & 4 & 85.2 & 55.6 & 0 \\
\hline B(18.5-23.9) & 37 & 69 & 51.5 & 23.1 \\
\hline C(24.0-32.0) & 14 & 60.9 & 55.6 & 20.0 \\
\hline X2 & 5.618 & 0.207 & 0.605 \\
\hline P & 0.06 & 0.902 & \\
\hline * The P $₫ 0.05$ was considered statistically significant & & \\
\hline
\end{tabular}

We further analyzed and compared the embryo morphokinetic parameters with the BMI groups (Table 5). The results showed that the tPNa was statistically significant among all groups $(\mathrm{P}<0.05)$ with a positive correlation. It is interesting to note that no statistical differences for the other embryo morphokinetic parameters were found among $(P>0.05)$, but the t2PBe, tPNf, and t2 increased gradually with the BMI increase.

Table 5

Comparison of embryo morphokinetic parameters with the different BMI groups

\begin{tabular}{|c|c|c|c|c|c|}
\hline Group & $\mathbf{N}$ & t2PBe(h) & $\mathrm{tPNa(h)}$ & tPNf(h) & t2(h) \\
\hline$A(\nabla 18.5)$ & 4 & $2.67 \pm 0.60$ & $5.28 \pm 0.96$ & $22.12 \pm 2.30$ & $24.77 \pm 2.24$ \\
\hline$B(18.5-23.9)$ & 37 & $2.82 \pm 0.93$ & $6.52 \pm 1.38$ & $23.44 \pm 3.09$ & $26.58 \pm 4.39$ \\
\hline$C(24.0-32.0)$ & 14 & $2.97 \pm 0.89$ & $6.54 \pm 1.47$ & $23.53 \pm 3.16$ & $26.63 \pm 3.67$ \\
\hline $\mathrm{F}$ & & 1.280 & 10.048 & 2.423 & 2.466 \\
\hline$P$ & & 0.279 & $₫ 0.001$ & 0.090 & 0.086 \\
\hline
\end{tabular}

The effect of embryo morphokinetic parameters during early development on the clinical outcome after embryo transfer

In this study, a total 46 fresh cycles of embryo transfer were carried out. Upon analysis of the pregnancy outcomes after embryo transfer, there were 19 patients from the ICSI cycles that were completely implanted (the number of gestational sacs matched with the number of transferred embryos) and 13 patients from the ICSI cycles that were not implanted (the biochemical marker for pregnancy was negative) and included in the analysis. Subsequently, all high-quality embryos from those patients were divided into the implantation group and non-implantation group. The average value of t2PBe, tPNa, tPNf, and t2 for the high-quality embryos from two groups, is presented in Table 6. The t2PBe $(2.68 \pm 0.82 \mathrm{vs} .3 .16 \pm 0.9, \mathrm{P}<0.05)$ and the tPNa $(6.31 \pm 1.11 \mathrm{vs}$. $7.06 \pm$ $1.32, P \llbracket 0.05)$ of the high-quality embryos were significantly shorter in the implantation group compared with those in the non-implantation group. Although no significant difference was found for the variables, tPNf and t2 between the implantation group and non-implantation group (Pख0.05), the tPNf and t2 were shorter in the implantation group.

Table 6

The effect of embryo morphokinetic parameters on the high-quality embryos from implantation or non-implantation patients after transfer

\begin{tabular}{|llllll|}
\hline Group & $\mathbf{N}$ & t2PBe(h) & tPNa(h) & tPNf(h) & t2(h) \\
\hline Implantation & 115 & $2.68 \pm 0.82$ & $6.31 \pm 1.11$ & $23.32 \pm 2.61$ & $25.92 \pm 2.64$ \\
\hline Non-implantation & 49 & $3.16 \pm 0.9$ & $7.06 \pm 1.32$ & $23.83 \pm 2.55$ & $26.55 \pm 2.57$ \\
T & & -3.217 & -3.767 & -1.144 & -1.406 \\
P & 0.002 & 0.000 & 0.254 & 0.162 \\
\hline * The P®0.05 was considered statistically significant & & \\
\hline
\end{tabular}

\section{Discussion}

Selection of embryos with the high developmental potential for embryo transfer to increase the implantation rate and thus the pregnancy rate is crucial in the ART program. The introduction of the TLM system successfully allowed the uninterrupted observation of the process of embryonic development and performed the dynamic assessment of early embryos. With the aid of the TLM system, the morphokinetic parameters of a single embryo can be effectively monitored. The morphokinetic parameters of the embryo during early development include the time taken for the second polar body extrusion (t2PBe), the time taken for the pronuclear appearance (tPNa), the time taken for the pronuclear fading (tPNf), and the time taken for the cleavage to 2-cells (t2), 3-cells (t3), 4-cells (t4) and 5-cells (t5) stages ${ }^{[9]}$. These embryonic developmental parameters can offer embryologists and clinicians the ability to effectively predict the potential of embryonic development and successful implantation. 
As early as 1997, Payne et al. (1997) used the time-lapse technology for the first time to monitor embryonic development including the time of polar body appearance, pronuclear appearance (tPNa), and pronuclear fading (tPNf). These parameters were found to be significantly different among different zygotes and also differed significantly between the high-quality embryos and low-quality embryos ${ }^{[13]}$. Subsequently, many studies recognized that the morphokinetic parameters of the embryo during early development could be used as key markers to predict the embryonic developmental potential.

In this retrospective study, we have found that the tPNf and t2 were significantly related to the embryo quality. Lemmen et al. (2008) found that the tPNa, tPNf, $\mathrm{t} 2, \mathrm{t} 3$, and $\mathrm{t} 4$ were related to the embryo quality and implantation potential, and the embryo development potential was greater when the t2 was shorter

${ }^{[14]}$. Barberet et al. (2019) concluded that the tPNf and t2 had significant correlations with the embryo quality, while the t2PBe and tPNa had not, and with relatively faster development, embryo quality was found to be high ${ }^{[10]}$. These conclusions are consistent with our results. Our results showed that the t2PBe and tPNa were not significantly different between the high quality and low-quality embryos, but the t2PBe and tPNa in the high-quality embryos are shorter than those in the low-quality embryos. Based on the analysis, the tPNf and t 2 in the present study were shorter by $1 \mathrm{~h}$ than the morphokinetic parameters reported by Coticchio et al. (2018) (tPNf: $23.36 \pm 3.08 \mathrm{~h}$ vs $24.5 \pm 4.7 \mathrm{~h}$, t2: $26.45 \pm 4.11 \mathrm{~h}$ vs $27.7 \pm 5.0 \mathrm{~h}$ ) ${ }^{[3]}$, and this difference may be related to the characteristics of the patients enrolled. We have found that the t2PBe, tPNa were not significantly related to the Day 3 embryo quality and the developmental potential, but the t2PBe $(2.68 \pm 0.82$ vs. $3.16 \pm 0.9, P \otimes 0.05)$ and the tPNa $(6.31 \pm 1.11$ vs. $7.06 \pm 1.32, P<0.05)$ of the high-quality embryos were significantly different between the implantation and non-implantation groups, indicating that the embryos with shorter t2PBe and tPNa have higher implantation potential. Meseguer et al. (2011) indicated that the t2, t3, t4, s2 (3-cells to 4-cells), and cc2 (2-cells to 3-cells) were significantly correlated with the embryo implantation potential, but their study did not evaluate the t2PBe, tPNa, and tPNf ${ }^{[15]}$. Carrasco et al. (2017) also showed that the morphokinetic parameters such as $\mathrm{t} 2, \mathrm{t} 3, \mathrm{t} 4$, and $\mathrm{t} 8$ could predict the embryo implantation ability, and the implantation ability was higher in the embryos with the faster cleavage rate, but the tPNa and tPNf were not significantly correlated with the embryo implantation ${ }^{[6]}$. Aguilar et al. (2014) revealed that the t2PBe and tPNf had a certain predictive value for embryo implantation ${ }^{[7]}$. Therefore, this current study analyzed the t2PBe, tPNa, tPNf, and t2, and found that the t2PBe, tPNa were significantly related to the implantation potential. Although the tPNf and t2 were not found to be significantly related to the implantation potential, median values of the tPNf and $\mathrm{t} 2$ in the high-quality embryos from the implantation group were shorter than those from the non-implantation group. These results were consistent with the outcomes of previous studies. The differences in conclusions may be due to the different embryo fertilization methods, culture environments, and population characteristics.

Some studies combined the duration of the cc2 and the duration of the 3-cells to 5-cells (cc3) in the Day 3 embryo morphology score to predict the blastocyst formation ${ }^{[16]}$. The results from Cruz et al. (2012) were consistent with this protocol, indicating that timing of cell division in human cleavage-stage embryos is linked with blastocyst formation and quality ${ }^{[17]}$. Desai et al. (2014) proposed that the morphokinetic parameters such as tPNf, t2, t4, and t8 could be used as indicators to predict the formation of the blastocyst ${ }^{[18]}$. Nevertheless, some scholars believed that embryo morphokinetic parameters have no correlation with embryo implantation rate, clinical pregnancy rate and live birth rate ${ }^{[8,19]}$. In our study, the morphokinetic parameters were not found to be significantly related to the blastocyst formation potential. It may be related to small sample size, different culture system and so on.

At present, the impact of BMI on embryo quality and the pregnancy outcome is controversial. Some scholars proposed that the blastocyst formation rate in the females with the normal BMI was higher than that in the obese women, and the BMI was significantly correlated with the embryo quality [20]. However, some scholars suggested that the BMI had no effect on the embryo morphokinetic parameters and embryonic development ${ }^{[21]}$. Bartolacci et al. elucidated the correlation between the BMI and the embryo morphokinetic parameters using the time-lapse monitoring system and found that the embryo development in overweight or obese females was slower ( $\mathrm{t} 5$ and t8 were prolonged), and these morphokinetic parameters may be used to improve the selection of the high-quality embryos ${ }^{[22]}$. Therefore, the present study further analyzed the participants with different BMI and found that the Day 3 high-quality embryo ratio and the blastocyst formation rate were not statistically different between participants with different BMI $(P>0.05)$. The only statistical difference was found for the tPNa among different groups $(P<0.05)$. Perhaps, due to the small sample size in the present study, significant differences for the tPNf and 2 may not have been found between the groups, but it is interesting to note that both tPNf and t2 gradually increased with the increase of BMI. Similarly, no significant difference regarding the ratio of the high-quality embryo on the Day 3 was not found between patients with different BMI. But the ratio of the high-quality embryo on Day 3 gradually declined with the increase of BMI. This analysis also showed that the Day 3 high-quality embryo rate gradually declined when the tPNf and t2 were longer (Table 1). Therefore, we speculated that the BMI could impact the early embryo quality affecting embryo morphokinetic parameters, and the sample size needs to be increased to further verify this correlation.

\section{Conclusions}

In summary, embryo morphokinetic parameters may be effectively used to predict the embryo quality to improve the selection of embryos in clinical practice. The embryos with a relatively faster development rate will have a greater high-quality embryo rate and implantation rate. This study included a relatively small sample and the morphokinetic parameters were affected by many factors. Therefore, more ICSI cycles need to be analyzed, and the strict inclusion criteria should be included to further explore the predictive value of the embryo morphokinetic parameters to determine the embryo quality and speculate the pregnancy outcome. It is expected that the embryo morphokinetic parameters in conjunction with the currently used conventional morphologic parameters could improve the selection of embryos and improve the outcome of the ICSI procedure.

\section{Abbreviations}

ART: assisted reproductive technology

TLM: time-lapse monitoring system 
2PB: second polar body

ICSI: intracytoplasmic sperm injection

t2PBe: time of second polar body extrusion

tPNa: time of pronuclear appearance

tPNf: time of pronuclear fading

t2: time of cleavage to 2-cells

rhCG: recombinant human chorionic gonadotropin

BMI: body mass index

AMH: anti-Müllerian hormone

\section{Declarations}

\section{Availability of data and materials}

All data generated through this study are included in this article.

\section{Acknowledgments}

Not applicable.

\section{Funding}

This study was supported by the National Natural Science Foundation of China (U1904138) and the National Key R\&D Program of China (2019YFA0110900).

\section{Authors' contributions}

QH and GDY design subject, analyze data and write manuscript. JH and GY analyze data and write manuscript. ZW, HF, YB, JZ, TZ, NS, HJ, WS and SS provide data and modify manuscript. GDY and YS supervised the subject. All authors read and approved the final manuscript.

\section{Ethics declarations}

\section{Ethics approval and consent to participate}

This study was approved by the Biomedical Ethics Committee of the First Affiliated Hospital of Zhengzhou University, and written informant consent was obtained from the patients.

\section{Consent for publication}

Not applicable.

\section{Competing interests}

The authors declare that they have no conflicts of interest.

\section{References}

1. Baczkowski, T, Kurzawa R, Głabowski W. Methods of embryo scoring in in vitro fertilization[J]. Reprod Biol. 2004;4(1):5-22.

2. Alpha Scientists in Reproductive M, Embryology ESIGo. The Istanbul consensus workshop on embryo assessment: proceedings of an expert meeting[J]. Hum Reprod. 2011;26(6):1270-1283.

3. Coticchio G, Mignini Renzini M, Novara PV, et al. Focused time-lapse analysis reveals novel aspects of human fertilization and suggests new parameters of embryo viability[J]. Hum Reprod, 2018, 33(1):23-31.

4. Motato Y, de los Santos M, Escriba M, et al. Morphokinetic analysis and embryonic prediction for blastocyst formation through an integrated time-lapse system[J]. Fertil Steril. 2016;105(2):376-384.e379.

5. Petersen BM, Boel M, Montag M, et al. Development of a generally applicable morphokinetic algorithm capable of predicting the implantation potential of embryos transferred on Day 3[J]. Hum Reprod. 2016;31(10):2231-2244.

6. Carrasco B, Arroyo G, Gil Y, et al. Selecting embryos with the highest implantation potential using data mining and decision tree based on classical embryo morphology and morphokinetics[J]. J. Assist. Reprod. Genet., 2017, 34(8):983-990.

7. Aguilar J, Motato Y, Escriba MJ, et al. The human first cell cycle: impact on implantation[J]. Reprod Biomed Online. 2014;28(4):475-484. 
8. Goodman LR, Goldberg J, Falcone T, et al. Does the addition of time-lapse morphokinetics in the selection of embryos for transfer improve pregnancy rates? A randomized controlled trial[J]. Fertil Steril. 2016;105(2):275-285 e210.

9. Barberet J, Bruno C, Valot E, et al. Can novel early non-invasive biomarkers of embryo quality be identified with time-lapse imaging to predict live birth? [J]. Hum Reprod. 2019;34(8):1439-1449.

10. Barberet, J, Bruno, C, Valot, E, et al. Can novel early non-invasive biomarkers of embryo quality be identified with time-lapse imaging to predict live birth? [J]. Hum Reprod (Oxford, England). 2019;34(8):1439-1449.

11. Brinsden PR. Textbook of In Vitro Fertilization and Assisted Reproduction[J]. British Medical Journal. 1999;2(2807):667-668.

12. Gardner DK, Lane M, Stevens J, et al. Blastocyst score affects implantation and pregnancy outcome: towards a single blastocyst transfer. Fertil Steril. 2000;73(6):1155-8.

13. Payne, D, Flaherty, SP, Barry, MF, et al. Preliminary observations on polar body extrusion and pronuclear formation in human oocytes using time-lapse video cinematography. Hum Reprod. 1997;12:532-541.

14. Lemmen, JG, Agerholm, I, ZiebeKinetic, S. Kinetic markers of human embryo quality using time-lapse recordings of IVF-ICSI-fertilized oocytes. Reprod Biomed Online. 2008;17:385-391.

15. Meseguer M, Herrero J, Tejera A, et al. The use of morphokinetics as a predictor of embryo implantation[J]. Hum Reprod. 2011;26(10):2658-2671.

16. Conaghan J, Chen A, Willman S, et al. Improving embryo selection using a computer-automated time-lapse image analysis test plus day 3 morphology: results from a prospective multicenter trial[J]. Fertil Steril. 2013;100(2):412-419.e415.

17. Cruz M, Garrido N, Herrero J, et al. Timing of cell division in human cleavage-stage embryos is linked with blastocyst formation and quality[J]. Reprod Biomed Online. 2012;25(4):371-381.

18. Desai N, Ploskonka S, Goodman L, et al. Analysis of embryo morphokinetics, multinucleation and cleavage anomalies using continuous time-lapse monitoring in blastocyst transfer cycles[J]. Reprod. Biol. Endocrin. 2014;12:54.

19. Adolfsson E, Porath S, Andershed A. External validation of a time-lapse model; a retrospective study comparing embryo evaluation using a morphokinetic model to standard morphology with live birth as endpoint[J]. JBRA Assist Reprod. 2018; 22(3):205-214.

20. Comstock IA, Kim S, Behr B, et al. Increased body mass index negatively impacts blastocyst formation rate in normal responders undergoing in vitro fertilization[J]. J Assist Reprod Genet. 2015;32(9):1299-1304.

21. Bellver J, Mifsud A, Grau N, et al. Similar morphokinetic patterns in embryos derived from obese and normoweight infertile women: a time-lapse study[J]. Hum Reprod. 2013;28(3):794-800.

22. Bartolacci A, Buratini J, Moutier C, et al. Maternal body mass index affects embryo morphokinetics: a time-lapse study[J]. J Assist Reprod Genet. 2019;36(6):1109-1116.

\section{Figures}

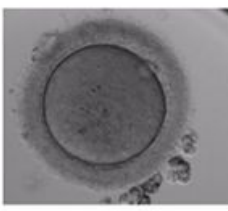

t0

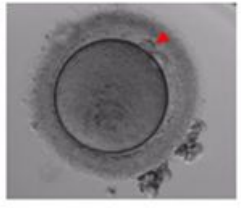

t2PBe

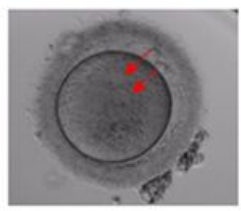

tPNa
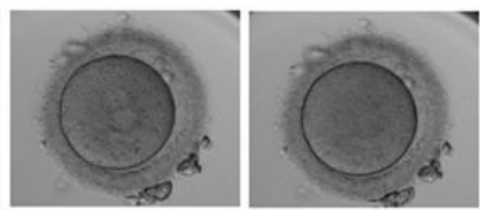

tPNf

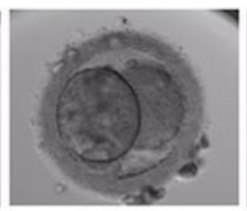

t2

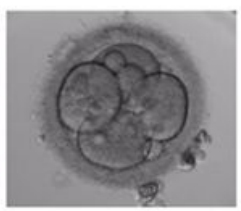

t4

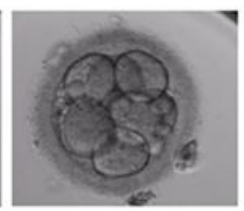

t8

Figure 1

The morphokinetic parameters of the embryos during the early embryonic development monitored using a time-lapse monitoring system in the ICSI cycle. The t0 refers to the time of ICSI completed, t2PBe refers to the time interval from the ICSI to the second polar body extrusion, tPNa refers to the time interval from the ICSI to the pronuclear appearance, tPNf refers to the time interval from the ICSI to the pronuclear fading, and t2 refers to the time interval from the ICSI injection to the cleavage of the fertilized zygotes to 2-cell embryos. The t4 and t8 refer to the time interval from the ICSI to the cleavage of the fertilized zygotes to 4- and 8-cell embryos, respectively. The red arrowhead points to the second polar body, and the red arrow points to the pronucleus. 